\title{
Physiological Arousal, Distress Tolerance, and Social Problem-Solving Deficits Among Adolescent Self-Injurers
}

\author{
Matthew K. Nock and Wendy Berry Mendes \\ Harvard University
}

\begin{abstract}
It has been suggested that people engage in nonsuicidal self-injury (NSSI) because they (a) experience heightened physiological arousal following stressful events and use NSSI to regulate experienced distress and (b) have deficits in their social problem-solving skills that interfere with the performance of more adaptive social responses. However, objective physiological and behavioral data supporting this model are lacking. The authors compared adolescent self-injurers $(n=62)$ with noninjurers $(n=30)$ and found that self-injurers showed higher physiological reactivity (skin conductance) during a distressing task, a poorer ability to tolerate this distress, and deficits in several social problem-solving abilities. These findings highlight the importance of attending to increased arousal, distress tolerance, and problemsolving skills in the assessment and treatment of NSSI.
\end{abstract}

Keywords: self-harm, self-mutilation, physiological arousal, distress tolerance, problem solving

Nonsuicidal self-injury (NSSI), which refers to the direct and deliberate destruction of one's own body tissue in the absence of intent to die and outside the context of socially or medically sanctioned procedures (e.g., ear piercing), is reported to occur in approximately 4\% of adults (Briere \& Gil, 1998; Klonsky, Oltmanns, \& Turkheimer, 2003) and 14\%-21\% of adolescents (Ross \& Heath, 2002; Whitlock, Eckenrode, \& Silverman, 2006; Zoroglu et al., 2003). Despite the seriousness and prevalence of NSSI, it continues to be a perplexing clinical problem, as it remains unclear why some individuals intentionally and repeatedly inflict harm on themselves.

Authors have theorized about the causes of NSSI for years (e.g., Menninger, 1938); however, systematic research has addressed this topic only more recently. Studies examining the proposed functions of self-injury suggest that individuals engage in such behaviors primarily (a) for affect regulation-most often to decrease or escape from extreme negative affect or aversive arousal-and (b) for social communication-such as to get attention from others or to influence their behavior in some way. These functions have been demonstrated among both adolescent (Nock \& Prinstein, 2004, 2005) and adult (Brown, Comtois, \& Linehan, 2002) samples of those engaging in NSSI as well as in a rich literature on NSSI among those with developmental disabilities (Durand \& Crimmins, 1988; Iwata et al., 1994). This earlier work has provided useful initial information about the processes that

Matthew K. Nock and Wendy Berry Mendes, Department of Psychology, Harvard University.

This research was supported by National Institute of Mental Health Grant MH076047 as well as by grants from the Milton Fund and Talley Fund of Harvard University to Matthew K. Nock. We thank members of the Laboratory for Clinical and Developmental Research for their assistance with this work as well as the participants in this study. We are grateful to Mitch Prinstein for his valuable help in devising the Distress Tolerance Test.

Correspondence concerning this article should be addressed to Matthew K. Nock, Department of Psychology, Harvard University, 33 Kirkland Street, Cambridge, MA 02138. E-mail: nock@wjh.harvard.edu may be involved in the etiology and maintenance of NSSI but has been limited by a general reliance on self-report, as individuals often are not able to adequately and accurately report on the forces influencing their own behavior (e.g., Nisbett \& Wilson, 1977). Nevertheless, prior research points toward several processes believed to play a role in the maintenance of NSSI that could be more carefully tested in subsequent studies, such as physiological hyperarousal, poor distress tolerance, and impairments in social problem-solving skills. The current study was designed to provide an initial, objective test of the relation of each of these three constructs to NSSI.

\section{Physiological Reactivity and NSSI}

The most commonly proposed explanation of NSSI is that self-injurers experience extreme and intolerable arousal in response to stressful events and engage in NSSI because doing so leads to cessation of this arousal (via distraction, endorphin release, or some other, as yet unknown mechanism), thus causing NSSI to be negatively reinforced. Prior studies have demonstrated that self-injurers report higher levels of subjectively experienced emotional distress in response to stressful events (Najmi, Wegner, \& Nock, 2007; Nock, Wedig, Holmberg, \& Hooley, in press) and also have demonstrated that imagining that one is engaging in NSSI decreases physiological arousal among self-injurers (Haines, Williams, Brain, \& Wilson, 1995). However, no studies have provided objective evidence of increased reactivity to stressful events among nonsuicidal self-injurers. This is not merely an academic point but represents an important gap in the research.

Work in related areas, such as the study of suicidal self-injury and borderline personality disorder, which both overlap with but are distinct from NSSI (e.g., Nock, Joiner, Gordon, LloydRichardson, \& Prinstein, 2006; Nock \& Kessler, 2006; O'Carroll, Berman, Maris, \& Moscicki, 1996), has failed to find consistent differences between these clinical groups and control participants on objective, peripheral physiological measures (e.g., skin conductance [SC]; Crowell et al., 2005; Ebner-Priemer et al., 2005; 
Edman, Asberg, Levander, \& Schalling, 1986; Herpertz, Kunert, Schwenger, \& Sass, 1999; Herpertz, Werth, et al., 2001). Notably, one recent study reported that although "parasuicidal" adolescent girls (a group combining suicidal and nonsuicidal self-injurers) did not differ from participants in a comparison condition on SC in response to a negative mood induction, the former group did show greater respiratory sinus arrhythmia activity (Crowell et al., 2005). In addition, several recent studies have reported amygdala hyperreactivity among women with borderline personality disorder relative to controls (Donegan et al., 2003; Herpertz, Dietrich, et al., 2001). Taken together, these findings suggest that although individuals with NSSI have a more aversive subjective emotional experience, there is mixed evidence of hyperarousal among those with related conditions such as borderline personality disorder, and there is currently no evidence of heightened peripheral physiological arousal among those engaging in NSSI.

Determining whether individuals who engage in NSSI truly experience increased physiological reactivity in response to stressful events is important not only for understanding this behavior problem but also for the purposes of assessment and treatment. Information about physiological response style has proven to be an important component in the understanding and treatment of other conditions, such as anxiety disorders, and some of the most efficacious treatments now incorporate psychoeducational materials early in the course of treatment to facilitate greater client understanding of and response to physiological reactivity (e.g., Barlow $\&$ Craske, 2000). The achievement of a greater understanding of the psychophysiology of NSSI may similarly lead to improvements in the treatment of NSSI.

There is a strong empirical basis for studying physiological arousal as indexed by changes in skin conductance level (SCL; Dawson, Schell, \& Filion, 2000). The physiological basis of SC includes measuring changes in eccrine (sweat) glands, which are innervated by the sympathetic branch of the autonomic nervous system via acetylcholine. An advantage of SC responses is that, unlike other responses associated with the autonomic nervous system, "individual differences in [SC] are most reliably associated with psychopathological states" (Dawson et al., 2000, p. 211). In general, inferences of SC changes are varied and generally nonspecific. For example, psychological states such as arousal, attention, excitement, fear, and anger all have been linked to SC changes. However, one can increase the psychological inferences of SC changes by examining changes within specific and welldefined contexts. In the current study, we use SCL as a general index of sympathetic nervous system arousal during a frustrating and distressing task in the context of an attention task.

\section{Distress Tolerance and NSSI}

A key assumption of the affect regulation model presented above is that self-injurers are less able (or less willing) to tolerate intense distress than noninjurers, regardless of whether the experience of greater reactivity is subjective or physiologically based, and that they use NSSI as a means of escaping from the experience of intense distress. This lack of distress tolerance is widely held to be an important explanatory factor in the development and maintenance of NSSI (Chapman, Gratz, \& Brown, 2006; Favazza, 1996; Klonsky, 2007). It is surprising, however, that no objective behavioral test of distress tolerance among self-injurers has been conducted. There are significant clinical implications for such a test, as improving distress tolerance is a key focus in commonly used treatments for NSSI (e.g., Linehan, Armstrong, Suarez, Allmon, \& Heard, 1991; Linehan et al., 2006). The demonstration that self-injurers actually have a problem tolerating distress would support this treatment focus, and the development of a behavioral measure of distress tolerance could be useful for measuring and studying potential mechanisms of change in treatment (Kazdin \& Nock, 2003; Lynch, Chapman, Rosenthal, Kuo, \& Linehan, 2006). In addition, information about how increased distress and an inability to tolerate such distress interact with other cognitive processes could be used to further inform and enhance such treatments.

\section{Problem Solving and NSSI}

Clinicians and researchers have focused primarily on the affectregulating properties of NSSI (e.g., Chapman et al., 2006; Favazza, 1989; Klonsky, 2007), with much less attention given to the social functions of this behavior. This is likely due to the fact that prior work on the functions of NSSI suggests that people most often engage in this behavior to regulate their affect (e.g., Nock \& Prinstein, 2004). However, it is important to bear in mind that experimental research on the functions of NSSI among developmentally disabled samples suggests that social reinforcement is the primary motivator of this behavior in this group (Iwata et al., 1994). In addition, a significant portion of adolescent (Nock, Holmberg, Photos, \& Michel, 2007; Nock \& Prinstein, 2004, 2005) and adult (Brown et al., 2002) self-injurers without developmental disabilities report engaging in NSSI to influence their environment in some way.

A related and fairly extensive literature has demonstrated that deficits in social problem-solving skills are related to suicide ideation and attempts among adults (Schotte, Cools, \& Payvar, 1990; Williams, Barnhofer, Crane, \& Beck, 2005) and among children and adolescents (Orbach, Rosenheim, \& Hary, 1987; Pollock \& Williams, 1998, 2001; Sadowski \& Kelley, 1993). This work has shown that suicidal individuals generate fewer and less effective solutions to social problems than those who are nonsuicidal and that these differences are not explained by IQ or the presence of other psychological disorders, such as depression (Biggam \& Power, 1999; Pollock \& Williams, 2001; Williams et al., 2005). Although valuable, this earlier research is limited by the fact that it did not examine NSSI, and the range of problem-solving deficits explored has been relatively narrow. While work on problem solving among suicidal individuals has primarily examined individuals' ability to generate adaptive solutions, research from other areas of psychological science has investigated a much broader range of potential deficits and dysfunctions.

Myriad deficits or dysfunctions can occur in the informationprocessing sequence that can influence engagement in maladaptive behaviors, such as problems with cue interpretation, response selection, and response enactment (see Crick \& Dodge, 1994; Ingram, 1986; McFall, 1982). It would be instructive to know whether and how such processes may be different among those engaging in NSSI. For instance, early in the informationprocessing sequence, self-injurers might make more self-critical attributions about the behavior of others (cf. Dodge \& Frame, 1982), which could lead to engagement in NSSI as a means of 
self-punishment. It is also possible that those engaging in NSSI generate fewer or less effective solutions than noninjurers, as has been shown to be the case in some studies of suicidal individuals (e.g., Schotte \& Clum, 1987), although not others (e.g., Sadowski $\&$ Kelley, 1993). Different still, regardless of the solutions generated, it may be that self-injurers select less effective responses from among those generated. In other words, they may, in fact, be able to generate numerous and effective solutions but then select less adaptive responses for behavioral enactment. The decision about which solution is selected and performed may be influenced by self-injurers' beliefs about their self-efficacy for effectively performing an adaptive solution. Although the investigation of comprehensive information-processing and problem-solving models has led to significant advances in the understanding of several different forms of psychopathology, as noted above, such models have not been used to examine NSSI.

The purpose of the current study was to conduct an initial test of these three related components of the previously examined functional model of NSSI (Nock \& Prinstein, 2004, 2005). Specifically, we examined whether, relative to noninjurious adolescents, adolescents with a history of NSSI demonstrate (a) heightened physiological reactivity in response to a stressful event, (b) an impaired ability or willingness to tolerate distress, and (c) deficits in the social problem-solving skill domains described above. Support for each of these hypotheses would advance understanding of why adolescents engage in NSSI and would have significant implications for work on the assessment and treatment of these behaviors.

\section{Method}

\section{Participants}

Participants $(N=92)$ were 62 adolescents and young adults (ages 12 to 19 years) with a history of engaging in NSSI and 30 noninjurious controls matched on age, sex, and race/ethnicity (Table 1). Two additional adolescents with a history of NSSI were recruited but excluded from analyses because of technical difficulties during data collection. We focused on adolescence and young adulthood given the significantly increased risk of selfinjurious thoughts and behaviors during this developmental period (Kessler, Borges, \& Walters, 1999; Nock \& Kazdin, 2002). All participants were recruited via study advertisements placed in local psychiatric clinics, in newspapers, on community bulletin boards, and on the Internet. The announcements for both control and self-injurious participants indicated,

We are seeking adolescents between the ages of 12-19, and their parents, to participate in a study aimed at understanding self-harm behaviors. Eligible participants will be paid for participation in this confidential study. Participation involves completing interviews, questionnaires, and computer tasks.

All participants who responded to the advertisement were invited to the laboratory and provided with a complete description of the study, and written informed consent was obtained, with parental consent obtained for participants younger than 18 years.

Although this sample was recruited from the community, many individuals reported that they were currently receiving psychological treatment (48.2\%) and/or pharmacotherapy (46.3\%), and most (76.6\%) met criteria for at least one current psychiatric disorder according to semistructured diagnostic interview (Kaufman, Birmaher, Brent, Rao, \& Ryan, 1997). The most common diagnoses were anxiety disorders (46.7\%), mood disorders (32.6\%), alcohol and substance use disorders $(14.1 \%)$, impulse-control disorders $(10.9 \%)$, and eating disorders $(6.5 \%)$, with an average of 2.0 $(S D=2.0)$ current disorders for the entire sample.

\section{Assessment}

Demographic factors. Participants provided information about demographic characteristics, including age, sex, and race/ethnicity, via face-to-face interviews. To ensure that any between-groups differences on the distress tolerance and problem-solving tests were not due to differences in IQ, we also assessed all participants using the Wechsler Abbreviated Scales of Intelligence (WASI; Wechsler, 1999).

NSSI. All participants were administered the Self-Injurious Thoughts and Behaviors Interview (SITBI; Nock, Holmberg, et al., 2007), a structured interview used to assess the presence, frequency, severity, age of onset, and other characteristics of a broad range of self-injurious thoughts and behaviors, including NSSI. Participants in the current study were classified on the basis of their responses to questions from the NSSI module of the SITBI

Table 1

Characteristics of the Participant Groups

\begin{tabular}{lcccc}
\hline \multicolumn{1}{c}{ Variable } & $\begin{array}{c}\text { NSSI } \\
(n=62)\end{array}$ & $\begin{array}{c}\text { Control } \\
(n=30)\end{array}$ & Range & Statistic \\
\hline Mean (SD) age in years & $17.4(1.8)$ & $16.7(2.0)$ & $12-19$ & $t(90)=1.66$ \\
Gender (\% female) & 79.7 & 73.3 & & $\chi^{2}(1)=0.48$ \\
Race/ethnicity (\%) & 75.0 & 70.0 & & $\chi^{2}(5)=3.30$ \\
$\quad$ European American & 3.1 & 3.3 & & \\
African American & 7.8 & 3.3 & & \\
Hispanic & 4.7 & 6.7 & & \\
Asian & 9.4 & 13.3 & & \\
Biracial & 0.0 & 3.3 & & \\
Other & $108.9(13.5)$ & $110.9(11.3)$ & $81-137$ & \\
Mean $(S D)$ Full Scale IQ & & & & \\
\hline
\end{tabular}

Note. $\quad$ NSSI $=$ nonsuicidal self-injury. 
about lifetime engagement in NSSI (e.g., "Have you ever purposely hurt yourself without intending to die?"). All participants with a lifetime history of NSSI were classified in the NSSI group, and those with no such history were classified in the noninjuring control group. The SITBI has strong interrater reliability (average $\kappa=.99$ ), test-retest reliability over a 6-month period (average $\kappa=$ .70 ), and construct validity, as demonstrated by strong relations with other measures of self-injurious thoughts and behaviors (Nock, Holmberg, et al., 2007).

The SITBI also assesses the self-reported function of NSSI via four questions (rated 0-4) inquiring about the extent to which the participant has engaged in NSSI for the purposes of (a) decreasing aversive thoughts or feelings (i.e., automatic negative reinforcement; ANR), (b) increasing positive thoughts or feelings (i.e., automatic positive reinforcement), (c) decreasing or escaping from social interactions (i.e., social negative reinforcement), or (d) increasing social interactions or access to resources (i.e., social positive reinforcement). These items were selected on the basis of prior research demonstrating these are the most commonly reported functions of NSSI (Durand \& Crimmins, 1988; Nock \& Prinstein, 2004, 2005), and each correlates strongly with longer measures of each function (Lloyd, Kelley, \& Hope, 1997; Nock \& Prinstein, 2004; $r \mathrm{~s}=.64$ to .73 ), supporting the validity of using these individual items.

Physiological arousal. Skin conductance data were collected during the distress tolerance and problem-solving portions of the laboratory session (described below) with Biopac (Goleta, CA) TSD203 transducers placed on the distal phalanges of the middle and ring fingers of the participant's nondominant hand. The experimenter abraded the skin on the fingers using a mild abrasive brush and then filled the transducers with electrode paste. Data were amplified with a GSR 100C amplifier (Biopac) with a gain of $10 \mu$ Siemens and a low-pass filter of $10 \mathrm{~Hz}$. Once data were collected, they were scored offline with Mindware's (2005) EDA 2.1 computer program in 1-min epochs. The software program calculates tonic SCL as the average response in the identified time epoch. All SC values are reported in microsiemens.

Distress tolerance. The ability to tolerate distress was assessed with a behavioral task developed for the current study. The Distress Tolerance Test (DTT) was administered via the stimulus cards from the Wisconsin Card Sort Test (WCST; Grant \& Berg, 1948; Heaton, Chelune, Talley, Kay, \& Curtis, 1993), and, as in the WCST, four key cards were dealt face up on the table and the standard WCST instructions were read, indicating that the participant was to match cards from a deck to the key cards. The examiner stated that she could not tell the participant how to match the cards but would indicate whether each card placed was correct or incorrect. Participants were then told that there were 64 cards in the deck, that they had to get through the first 20 of them, and that it was up to them how far to continue beyond that point. Regardless of where the participant placed the cards, the examiner responded "correct" to the first 3 cards (to engage the participant in the task) and "incorrect" to the next 7 (to induce distress). The 11th card was "correct" (to reengage the participant), and all remaining cards were "incorrect," with a brief pause for mood rating after the 20th card. Prior studies have used similar card sorting tasks to induce experimental distress (e.g., Hirito \& Seligman, 1975; Ruggero \& Johnson, 2006). The DTT builds on this earlier work by providing more consistently negative feedback over a smaller number of trials (thus serving as a more "compact" distress induction) and by including the opportunity to escape after 20 trials, which provides a behavioral measure of distress tolerance.

Pilot testing of the DTT among laboratory staff unfamiliar with the DTT and the study hypotheses revealed that individuals completing the task consistently reported experiencing frustration during this task. In addition, self-report data collected after the 20th card from participants in the current study as a manipulation check further supported this, with participants reporting significantly more negative affect (i.e., sum of "frustrated," "angry," and "confused," each rated on a $0-4$ scale) than positive affect (i.e., total of "happy," "confident," and "satisfied"), $t(65)=6.37, p<.001$. Total score on the DTT was indexed by the number of cards for which the participant persisted at this task. It was inferred that those who persisted at this task despite repeated failure had greater distress tolerance.

Social problem-solving skills. Social problem-solving skills were assessed with a novel performance-based task called the Social Problem-Solving Skills Test (SPST; Nock, 2006). Measures exist that assess a broad range of problem-solving skills using a person's self-report (e.g., D'Zurilla \& Nezu, 1990) or that assess a specific problem-solving skill (e.g., generation of potential responses to a problem) using behavioral performance (e.g., Platt, Spivack, \& Bloom, 1975). The SPST was designed to build on these earlier tasks by measuring a broad range of problem-solving skills on the basis of behavioral performance. Drawing on prior work from other areas that has used multicomponent, performance-based measures of problem-solving skills (e.g., Dodge \& Somberg, 1987; Goddard \& McFall, 1992), the SPST asked participants to listen to a series of audio recordings describing eight social scenarios in four different domains (i.e., two scenarios in each domain) involving potential problems with peers (e.g., "You walk into a local pizzeria to meet your friends. As soon as you walk in, one of them says: 'Hey, look who it is!' and they all start laughing"), a boyfriend or girlfriend (e.g., "You are out to dinner on a Saturday night with your boyfriend. For the third time this week you notice him staring at a really pretty girl while you are talking to him about something really important to you"), a parent (e.g., "You're beginning to make friends with some really cool people. They tell you about an amazing party this weekend that you have to go to. You go home and tell your mother about it and she says you can't go"), and a teacher or boss (e.g., "You worked really hard on an English paper, a personal essay about what you admire about yourself. Your teacher hands it to you and you got a $\mathrm{C}-$. The major criticism is that you weren't specific enough"). After hearing each scenario, the participants performed various problem-solving tasks that examined different facets of their social problem-solving abilities. Their performance on each part of the SPST was recorded via video and audio tape and subsequently scored by two independent, blind raters. The raters followed a manualized coding system (Nock, 2006), and analysis of 30 randomly selected cases revealed adequate interrater reliability for each construct assessed (described below).

After each scenario, participants first were asked to describe in their own words why the antagonist in each situation behaved the way he or she did. Their attributions were coded by the blind raters as either self-critical (e.g., "Because I am ugly"), critical of the antagonist (e.g., "Because he is a jerk"), or noncritical (e.g., "Because things sometimes just happen that way"; Number of 
Critical Attributions subscale; $\kappa=.68$ ). Second, we assessed participants' ability to generate multiple solutions to each problem by recording the number of different solutions they were able to generate in a 15-s time span (Response Generation subscale; $r=$ .88). Prior studies using similar methods have given longer periods of time to generate solutions (e.g., 2 min; Williams et al., 2005); however, we allowed this shorter period of time to increase the external validity of this test, as social problems such as those presented in the DTT often must be solved quite quickly, and it was this ability that we were interested in assessing.

Third, the quality of each of the solutions generated was coded on a 1-3 scale according to whether content was negative (1; e.g., "yell at him" or "starve myself"), neutral (2; e.g., "ignore it"), or positive (3; e.g., "talk to her about it"; Response Content subscale; $r=.77$ ). Fourth, participants were asked to select the response from those generated that they would be most likely to actually perform (Response Selection subscale; $r=1.00$ ), and we examined group differences in the content of the response selected. Fifth, participants were asked to rate how effective they believed they would be at performing a model response on a $0-4$ scale (Self-Efficacy subscale; $r=1.00$ ). Participants also were asked to act out a specific adaptive response presented to them by the interviewer, and their behavioral enactment was coded for clarity, assertiveness, and other specific response characteristics; however, coders did not reach an acceptable level of reliability in their coding of these categories, so these data are not reported here. Analyses supported the interrater reliability (reported as kappas and correlations) of the five problem-solving skills described above. The construct validity of the SPST also was supported, as evidenced by relations between scores on the adolescentcompleted Social Skills Rating System Social Skills subscale (Gresham \& Elliott, 1990) and the SPST Number of Critical Attributions $(r=-.34, p<.01)$, Response Selection $(r=.27$, $p<.05)$, and Self-Efficacy subscales $(r=.44, p<.001)$, although not the Response Generation $(r=.10, n s)$ or Response Content subscales $(r=-.04, n s)$.

\section{Procedures}

All data were collected during one laboratory visit, and all study procedures were approved by the Harvard University institutional review board. All potential participants received a description of the study procedures and provided informed consent or assent to participate. They were informed that participation was voluntary and they could discontinue at any time; however, no one presenting to the laboratory refused to participate, and no one withdrew from the study. In all cases, adolescents were interviewed and assessed without their parent present to maximize honest responding. All adolescents and parents were informed during the consent procedure that all information they provided would be kept confidential unless we learned during the course of the study that the adolescent, parent, or someone they knew was in danger of being seriously harmed. We further informed them that in such instances we would undertake whatever measures we believed necessary to ensure the safety of those involved, such as contacting the local hospital or informing the parent if we believed the adolescent's self-injury or suicidal thoughts or plans put him or her at imminent risk of serious harm.
All participants first completed the interviews and WASI. Following a brief break, participants were seated in a testing room and connected to the GSR recording equipment for a brief resting baseline period. They were then administered the SPST and DTT. We administered participants Scenarios 1-4 of the SPST, then the DTT, then Scenarios 5-8 of the SPST to test the influence of distress on problem-solving abilities among self-injurious individuals relative to controls. All of these procedures took approximately 3-4 hr to complete and were administered by Matthew K. Nock and several graduate students and research assistants trained in these procedures and closely supervised by him. After completion of the study, all participants were debriefed and informed of the deception and intentional distress involved in the DTT. There were no concerns or complaints expressed regarding these procedures, and in many cases the adolescents expressed relief in knowing that there was no correct solution to the DTT. We also completed a thorough risk assessment with each adolescent (regardless of NSSI status) to ensure that he or she did not leave the laboratory in a state of distress and also to ensure that adolescents and parents were aware of the adolescents' current level of risk and to provide clinical referrals if needed. All participants were paid $\$ 100$ for their participation in this study.

\section{Data Analysis}

All variables were examined prior to analyses for normality and the presence of outliers, and in several cases variables were transformed and outliers assigned values one unit higher than the next most extreme score to reduce their influence (Tabachnick \& Fidell, 2001). In each case, the transformed variables more closely approximated a normal distribution, as measured by the ShapiroWilks normality test (Shapiro \& Wilks, 1965). We conducted preliminary analyses ( $t$ tests and chi-square tests) to compare those with a history of NSSI to control participants on demographic factors and IQ to ensure equivalence between groups on these key matching variables.

To examine whether those engaging in NSSI showed greater physiological reactivity than noninjurers (Hypothesis 1), we compared these two groups on changes in SCL by taking the baseline SCL (taken before the participant began the SPST) and subtracting that value from each minute of the DTT period. The DTT was designed so that participants could persist or quit after the first 20 trials/cards, resulting in progressively fewer participants across the course of the task. To allow for varying observations, we used multilevel modeling, which allows for missing data on any occasion without excluding participants like repeated measures analysis of variance. A multilevel approach takes advantage of all available data to generate parameter estimates. In this case, Level 1 consisted of the repeated assessment of SC changes during the frustration task, and Level 2 was the participant. Group differences (NSSI or control) were specified as a fixed effect. We used age and handedness of participant as covariates because of their wellestablished relationship to SC (Dawson et al., 2000). To examine whether those engaging in NSSI had poorer distress tolerance than noninjurers (Hypothesis 2) and whether self-injurers also showed impairments in their abilities for social problem solving (Hypothesis 3), we tested between-groups differences on the DTT as well as each of the SPST subscales with $t$ tests for independent samples, using the transformed variables. Untransformed scores on the DTT 
and SPST are reported in the Results section to facilitate interpretation of the study findings. ${ }^{1}$ All tests were two-tailed, with alpha set at .05 .

\section{Results}

\section{Preliminary Analyses}

Participants with a history of NSSI did not differ from controls on age, sex, ethnicity, or Full Scale IQ as measured by the WASI, as presented in Table 1, which indicates that any group differences observed in subsequent analyses are not attributable to these factors. Among the 62 adolescents with a lifetime history of NSSI, all reported at least two episodes of NSSI, 56 (90.3\%) reported engaging in NSSI within the past year, and 45 (72.6\%) reported doing so within the past month. The characteristics of NSSI in this sample were consistent with, but slightly less severe than, those reported in prior studies of NSSI among inpatient adolescent self-injurers (Nock \& Prinstein, 2004, 2005). In the current sample, the average age of onset for NSSI was 13.5 years $(S D=2.7)$, and the average number of episodes of NSSI in the past year (with a maximum set at 500 to reduce the influence of extreme outliers) was $62.6(S D=130.9, M d n=12.5) .{ }^{2}$ Self-injurers in this sample used commonly reported methods of NSSI, including cutting (90.6\%), scraping skin (51.6\%), self-hitting (51.6\%), and burning $(40.6 \%)$. Most self-injurers $(92.2 \%)$ had used more than one method in the past $(M=3.2, S D=2.9)$.

\section{Physiological Reactivity Among Self-Injurers}

Our first hypothesis was that self-injurers would exhibit significantly greater increases in physiological arousal (indexed by SC changes) than noninjurers during a distressing task. In support of this hypothesis, analyses revealed a significant effect for group, $F(1,81)=6.61, p<.05$ (Cohen's $d=0.57$ ). This represents a medium effect size. Adjusted means are plotted in Figure 1. As can be seen, the NSSI group exhibited greater changes in SCL over time than the control group, and this difference became especially pronounced in the later minutes of the DTT, when participants were informed that their answers were consistently incorrect. We also conducted these analyses with other diagnoses that might correlate with SC as covariates-major depressive disorder, posttraumatic stress disorder, and attention-deficit/hyperactivity disorder-and the results remained even after we accounted for other diagnoses, $F(1,78)=4.43, p<.05$.

Building on these findings, we then examined whether physiological reactivity was especially strong among self-injurers who reported engaging in NSSI for the purpose of decreasing aversive arousal. Our specific prediction was that individuals who reported that they engaged in NSSI for ANR on the SITBI (i.e., "To what extent do you engage in NSSI to get rid of bad feelings?" reported on a $0-4$ scale) would exhibit greater physiological reactivity during the DTT, further supporting the emotion regulation model of NSSI. To test this prediction, we averaged SCL across the entire DTT task. The ANR variable was not normally distributed ( $W=$ $0.82, p<.001$ ) and was obtained with a single item from the SITBI. Given the nonparametric nature of this variable, we used Spearman rank-ordered correlations to examine the relationship between ANR and SC changes. As predicted, greater endorsement of the ANR question was associated with greater increases in SC during the frustration task, although this medium-sized effect was just short of statistical significance (Spearman $r=.25, p=.055$ ).

\section{Distress Tolerance Among Self-Injurers}

Our second hypothesis was that self-injurers would show poorer distress tolerance than noninjurers, in that they would elect to stop the DTT earlier than noninjuring controls. Consistent with this hypothesis, self-injurers persisted at the DTT for significantly fewer cards $(M=26.3, S D=12.6)$ than noninjurers $(M=33.1$, $S D=16.1), t(90)=2.47, p<.05(d=0.52)$. This difference represents a medium effect size. There were no differences on the DTT between those in the high-ANR group $(M=26.6, S D=$ 13.7) versus the low-ANR group $(M=25.4, S D=8.8), t(60)<$ $0.75(d=0.19)$.

\section{Social Problem-Solving Skills Among Self-Injurers}

Our third hypothesis was that self-injurers would differ significantly from noninjurers on their abilities for social problem solving as measured by the SPST. Analyses revealed that self-injurers and noninjurers did not differ in the average number of self-critical attributions made, in the average number of solutions they generated in response to the challenging social situations, or in the average quality of solutions generated (Table 2). However, several important group differences were observed. That is, self-injurers chose significantly more negative solutions across the scenarios and rated their self-efficacy for performing adaptive solutions as significantly lower than that of noninjurers. These statistically significant findings represent medium to large effects, as presented in Table 2.

In an effort to understand how physiological arousal and social problem-solving skills might interact among these adolescents, we examined the extent to which the distress caused by the DTT interfered with problem solving, as it may be that problem-solving deficits are especially apparent during times of distress. Consistent with this notion, we found that, for the entire sample, the average number of solutions generated for each scenario decreased significantly from before $(M=3.7, S D=1.3)$ to after $(M=3.2, S D=$ $1.1)$ the DTT, $t(90)=6.18, p<.001(d=0.65)$. In addition, the number of other-critical attributions increased from before $(M=$

\footnotetext{
${ }^{1}$ Several authors have suggested that it is undesirable to perform parametric tests on transformed data, given that transformations can introduce other problems, such as altering the metric of the variable (e.g., Jaccard \& Guilamo-Ramos, 2002). To be sensitive to such issues, we conducted analyses on all of the DTT and SPST variables using parametric tests $(t$ tests for independent samples) on both untransformed and transformed variables, and we also conducted these analyses using nonparametric tests (i.e., Mann-Whitney $U$ tests). Each test yielded very similar results, in that there were only minor changes in effect size and no changes in significance tests. We therefore report test statistics and effect sizes from the parametric tests using transformed variables but report the untransformed means and standard deviations to facilitate interpretation of the findings.

${ }^{2}$ It is important to note that although there was significant variability in the frequency of NSSI in this sample, the study results were not driven by those engaging in high-frequency NSSI. In fact, lifetime frequency of NSSI was not significantly correlated with any of the primary outcome variables (e.g., DTT, SPST subscales, SC responses; $r \mathrm{~s}=-.07$ to .05 ).
} 


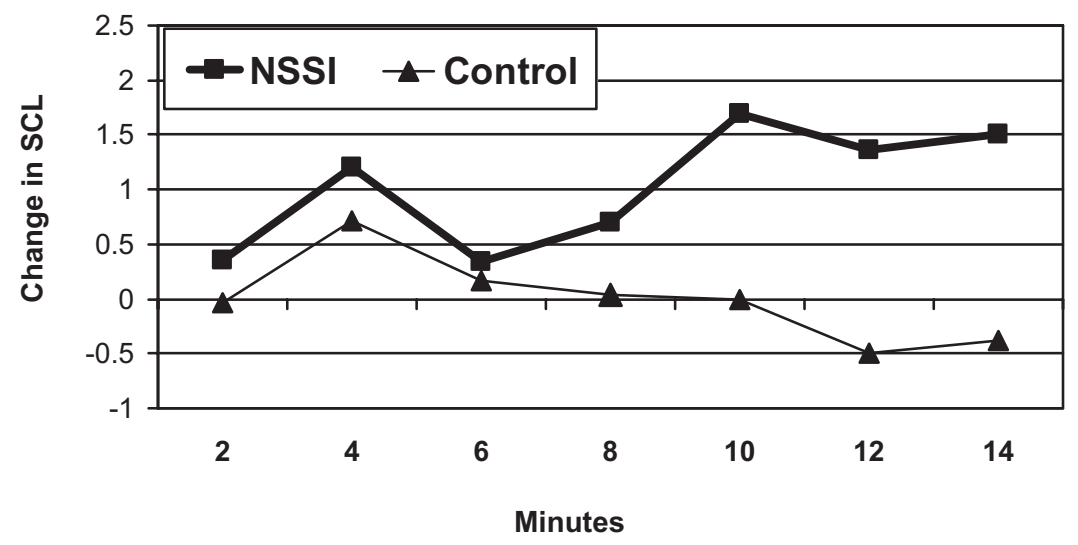

Figure 1. Changes in mean skin conductance level (SCL) during the Distress Tolerance Test. NSSI $=$ nonsuicidal self-injury.

$0.3, S D=0.5)$ to after $(M=0.7, S D=0.8), t(90)=-5.00, p<$ $.001(d=0.53)$, which was associated with a decrease in the number of self-critical attributions from before $(M=0.7, S D=$ $0.8)$ to after $(M=0.3, S D=0.5)$ the DTT, $t(90)=4.12, p<.001$ $(d=0.43)$. However, there were no significant group or Group $\times$ Time interaction effects for any of these measures, nor were there differences in the average quality of solutions generated, $t(90)=$ $0.60(d=0.06)$; quality of selected responses, $t(90)=0.50(d=$ $0.05)$; or report of self-efficacy, $t(90)=1.06(d=0.11)$.

\section{Discussion}

This study provided an objective test of several components of a theoretical model of NSSI that proposes that people engage in NSSI in response to extreme and intolerable emotional reactivity and as a result of deficits in social problem-solving skills (Nock \& Prinstein, 2004). In support of this model, we found that, compared to noninjurious adolescents, those with a history of NSSI displayed (a) increased physiological reactivity to a stressful task, (b) a decreased ability to tolerate distress and persist at this task, and (c) deficits in several specific social problem-solving skills. Several facets of these findings warrant more detailed comment.
This study provides the first evidence of physiological hyperarousal in response to a stressful event among those engaging in NSSI relative to noninjurers. Clinical reports have described increased arousal among self-injurers, and such arousal also has been suggested by the self-reports of self-injurers (Najmi et al., 2007; Nock, Wedig, et al., in press). This study provides physiological evidence to support this process, and the current data complement earlier evidence of a decrease in physiological arousal that occurs following script-driven imagery about engaging in NSSI (Haines et al., 1995). Although these studies have revealed evidence consistent with the emotion regulation theory of NSSI, several prior studies of related constructs, such as suicide attempts (Edman et al., 1986), "parasuicide" (which includes both suicidal and nonsuicidal self-injurers; Crowell et al., 2005), and borderline personality disorder (Herpertz et al., 1999; Herpertz, Werth, et al., 2001), have failed to find similar patterns of hyperarousal in the peripheral nervous system.

There are at least two explanations for the divergent findings. One possibility is that this hyperarousal is specific to NSSI. Both our study and that by Haines et al. (1995) focused specifically on NSSI, so this is a plausible explanation. However, this is unlikely

Table 2

Between-Groups Differences on Distress Tolerance and Problem-Solving Tests

\begin{tabular}{lccccc}
\hline \multicolumn{1}{c}{ Social Problem-Solving Skills Test } & Range & $M(S D)$ & $M(S D)$ & $t(90)$ & $\begin{array}{c}\text { Effect size } \\
(d)\end{array}$ \\
\hline $\begin{array}{l}\text { Attributions } \\
\quad \text { No. self-critical attributions } \\
\begin{array}{l}\text { Response Generation } \\
\quad \text { No. solutions generated }\end{array}\end{array}$ & $0-4$ & $1.1(1.1)$ & $0.9(1.0)$ & 0.70 & 0.15 \\
$\begin{array}{l}\text { Response Content } \\
\quad \text { Quality of overall solutions (coded 1-3) }\end{array}$ & $1.4-5.1$ & $3.3(0.9)$ & $3.5(0.8)$ & 0.75 & 0.16 \\
$\begin{array}{l}\text { Response Selection } \\
\quad \text { Quality of chosen solution (coded 1-3) }\end{array}$ & $1.4-3.0$ & $2.5(0.3)$ & $2.5(0.2)$ & 1.34 & 0.28 \\
$\begin{array}{l}\text { Self-Efficacy } \\
\quad \text { Self-efficacy rating (0-4) }\end{array}$ & $1.6-3.9$ & $2.5(0.5)$ & $3.0(0.4)$ & $4.28^{* * *}$ & 0.90 \\
\hline
\end{tabular}

Note. $\quad$ NSSI $=$ nonsuicidal self-injury.

${ }^{*} p<.05 .{ }^{* * *} p<.001$. 
given the overlap among all of these groups and the similarities in the subjective emotional experiences reported by these different groups. A more likely explanation is that the methods used in the current study, as well as that by Haines et al. (1995), were perhaps better suited to elicit and measure the hyperarousal experienced by self-injurers. For instance, prior studies have attempted to assess hyperarousal by showing a scene from a sad movie (Crowell et al., 2005) or by measuring immediate physiological responses to the brief (e.g., $6 \mathrm{~s}$ ) presentation of negative images (Herpertz, Werth, et al., 2001). In contrast, the current study used a frustrating task that required ongoing engagement, and it is interesting that the difference in arousal between self-injurers and noninjurers did not emerge until the 8 th min of the task, at which point self-injurers became increasingly aroused, while noninjurers showed a slight decrease in arousal. This suggests that the physiological hyperarousal experienced by self-injurers in response to stressful events may not be immediate (i.e., not within seconds of encountering a stressful situation) but rather increases after a brief period of frustration (e.g., after several minutes). If replicated, this finding will provide useful information to researchers and clinicians working with this population.

The validity of the hyperarousal findings in this study is further supported by the significant relation observed between physiological arousal and adolescents' self-report of engaging in NSSI for the purposes of ANR. Indeed, prior studies have consistently found that the majority of self-injurers report engaging in NSSI to decrease the experience of aversive hyperarousal. We found that those who reported engaging in NSSI to escape hyperarousal experienced the strongest physiological arousal during the distressing task. These findings provide further support for the selfreported functions of NSSI described in prior studies (Nock \& Prinstein, 2004, 2005).

Suicidal and nonsuicidal self-injury have long been proposed to function as a means of escape from intolerable emotional states (e.g., Baumeister, 1990; Favazza, 1996), and treatments for selfinjurers have included components that teach patients how to better tolerate distress (Linehan et al., 1991; Miller, Rathus, \& Linehan, 2007; Rudd, Joiner, \& Rajab, 2001). However, beyond obtaining self-reports of the reasons for engaging in these behaviors (Brown et al., 2002; Durand \& Crimmins, 1988; Hawton, Cole, O'Grady, \& Osborn, 1982; Nock \& Prinstein, 2004; Rodham, Hawton, \& Evans, 2004), there has been no test of whether self-injurers are actually more likely to have trouble tolerating or persisting in the face of distress and whether they attempt to escape from distressing situations more quickly than noninjurers.

This study provides the first objective evidence that self-injurers actually show decreased distress tolerance. It is possible that the difference observed on the DTT in the current study was not completely due to a lack of ability but also resulted from a decreased willingness to persist at this task. It is important to clarify this issue in future research, and investigators could do this by providing a desirable incentive for task persistence. Whether because of a lack of ability or will, the decreased distress tolerance and persistence observed among self-injurers are of scientific and clinical importance and are deserving of attention in future research and clinical efforts.

These findings of elevated physiological arousal and poor distress tolerance among those engaging in NSSI may be particularly useful to clinicians working with self-injurers as well as to selfinjurers themselves. The fact that self-injurers have an increased physiological response to stress may help clinicians, adolescents, and families better understand the experiences that may be driving NSSI and can inform treatment efforts and perhaps validating responses from family members. Moreover, these findings highlight the importance of focusing on distress tolerance skills in the treatment of NSSI (Linehan, 1993; Miller et al., 2007; Rudd et al., 2001).

The findings from this study also extend prior work examining the relation between social problem-solving skills and NSSI. Prior studies have revealed social problem-solving skills deficits among suicidal individuals (Sadowski \& Kelley, 1993; Schotte \& Clum, 1987; Schotte et al., 1990; Williams et al., 2005) and women with borderline personality disorder displaying parasuicide (Kehrer \& Linehan, 1996), and the current findings suggest such deficits also are present among those engaging in NSSI. It is interesting, however, that deficits in social problem-solving skills were not observed to be global in nature but instead were specific to several components of the problem-solving process, as described below.

Contrary to our hypotheses, self-injurers did not make more self-critical attributions than noninjurers. Thus, although children and adolescents who engage in aggressive behaviors make more hostile attributions toward others (Dodge \& Frame, 1982; Dodge \& Somberg, 1987), and prior work suggests that adolescents engaging in NSSI report being more self-critical than noninjuring adolescents (Glassman, Weierich, Hooley, Deliberto, \& Nock, 2007), the current study did not reveal a self-directed hostile attribution bias among self-injurious adolescents. It is possible that self-criticism among self-injurers is more general and does not necessarily occur in the context of problem solving or that our SPST task was not adequate to detect self-critical attributions (such an interpretation finds support in the relatively low number of self-critical statements made by both groups).

Self-injurious adolescents also did not show deficits in the quantity or quality of the solutions they generated to socially challenging situations. This suggests that, given time to think about a problem, self-injurers can produce effective solutions at the same level as noninjurers. However, self-injurers selected more maladaptive responses from those generated and reported lower self-efficacy for performing adaptive solutions, which might have influenced their response selection. Overall, these results provide a nuanced picture of the social problem-solving deficits selfinjurious adolescents may experience in everyday life.

It is interesting that, just as scores on the different components of the SPST were not uniformly associated with engagement in NSSI, they were not uniformly associated with a self-report measure of problem-solving skills either (i.e., Social Skills Rating System). In particular, the Response Generation and Response Content scores on the SPST were unrelated to both self-reported social skills and NSSI. One possible interpretation of the findings is that these two components of the SPST did not provide a valid measure of these abilities. This interpretation is difficult to support given that these two components were based directly on participants' actual performance and that our blind raters scored this performance with strong interrater reliability. Another interpretation is that the abilities to generate multiple and higher quality solutions are simply not related to engagement in NSSI and other problem behaviors. That is, perhaps all that is needed is the ability 
to generate one good solution to a difficult situation. This interpretation is consistent with the results observed here and, if replicated, would help focus the work of clinicians treating adolescents who engage in NSSI.

It also is important to note that although the overall number of solutions generated and the attributions made in each scenario changed from before to after the DTT, the problem-solving skills of self-injurers were no more impaired by the DTT than those of the noninjurers. This was surprising given prior research suggesting that cognitive abilities can be impaired through the experience of intense emotion (e.g., MacKay et al., 2004; Ruggero \& Johnson, 2006) and because self-injurers showed great physiological arousal on the DTT. It is possible that the distress induced by the DTT was different in quantity or quality from the type of distress that may trigger an episode of NSSI, and perhaps tasks that induced stronger or more personally salient distress would have led to differences in problem-solving abilities. In the current study, the presence of between-groups differences prior to the DTT indicates that selfinjurers have deficits in social problem-solving skills that do not occur only in the context of intense arousal but that are apparent even during times of relative calm.

Taken together, the findings on the social problem-solving skill deficits present among self-injurers provide valuable information about the aspects of social problem solving most likely to be involved in the decision to engage in NSSI, and they supply important information for future research and clinical efforts in this area. For instance, current treatments for NSSI include components focusing on improving social skills in general (Linehan et al., 1991; Miller et al., 2007). The current findings suggest that it may be most beneficial for clinicians to focus not on helping self-injurers learn how to generate more solutions but on helping them to select adaptive solutions for enactment. This may involve teaching self-injurers to slow down their problem-solving process to generate effective solutions and select the one most likely to be most effective, not merely the first one generated. This same clinical approach has proven effective in the treatment of child conduct problems (e.g., Kazdin, Siegel, \& Bass, 1992; Nock, 2003) and may be similarly beneficial in the case of NSSI. Moreover, the SPST (and perhaps the DTT) can be used in clinical and clinical research settings more generally, such as to test social problem-solving (and distress tolerance) skills among adolescents before, during, and after treatment to examine their abilities and improvements in these domains. This would not only be informative to the clinician, client, and family in each case but also could lead to significant advances in our understanding of the mechanisms of therapeutic change (Kazdin \& Nock, 2003; Lynch et al., 2006).

The findings from this study must be interpreted in the context of several important limitations, each of which points toward important directions for future research in this area. First, the current sample was relatively small and included adolescents, mostly female, who volunteered to participate in this study; therefore, our results may not generalize to other age groups or settings or to individuals unwilling to participate in clinical research. These results must be replicated in a larger, more diverse sample. In addition, it is important to highlight that the performance-based measures used in this study have not yet been validated on independent samples. A related point is that some of the assessments used were developed for use with adolescents (e.g., the scenarios presented in the SPST dealing with schoolwork and peer relations), and it is important to modify some aspects of these tasks when used with older samples.

Second, although our sample included only adolescents and young adults, the majority of whom had engaged in NSSI in the past month, there was some variability in the sample in terms of the timing, frequency, and severity of NSSI, and future research needs to consider such factors when examining the physiological and behavioral correlates identified in the current study. For instance, it is possible that the heightened physiological reactivity and poor distress tolerance described in the current study are present primarily among those engaging in severe and repetitive NSSI but less so among those who engage in NSSI one or two times or as a result of social modeling. It also is likely that some of these physiological and behavioral correlates of NSSI may become less pronounced following treatment or after a person stops engaging in NSSI, regardless of treatment history. These remain important questions for future research in this area.

Third, these data were cross-sectional and correlational in nature, limiting our ability to make inferences about the direction of the relations among study constructs. Our theoretical model suggests that adolescents engage in NSSI because of the physiological arousal, poor distress tolerance, and deficits in social problemsolving skills observed in this study; however, it is equally as likely that the differences observed somehow resulted from prior engagement in NSSI. This is less plausible, but prospective studies are needed to conclusively demonstrate the temporal relation between these constructs and NSSI. In addition, although performance on the SPST decreased following the DTT, because we did not randomly assign participants to the DTT condition, we cannot rule out the possibility that performance decreased simply because of fatigue or some other factor. Moreover, although the change in performance was statistically significant, the clinical significance of such a change is not clear from this initial test. In addition to prospective tests, the use of experimental manipulation is needed to further clarify these findings.

Fourth, we used only one method of measuring physiological arousal. It is important to expand on these measures in subsequent research and also to begin to examine these constructs outside the laboratory setting. As one example of such an effort, we are currently conducting a study that builds on the current findings by using ambulatory measurement of heart rate and respiratory sinus arrhythmia among those engaging in NSSI to examine the realtime physiological experiences of self-injurers. The ongoing examination of self-injurious thoughts and behaviors using multiple measurement methods in both the laboratory and real-world settings will significantly enhance our understanding of these behavior problems.

Fifth and finally, the model examined in this study was overly simple in nature and did not account for many of the factors likely to influence engagement in NSSI. For instance, the three constructs examined do not address the use of NSSI for automatic positive reinforcement (i.e., feeling generation) and say little about how NSSI may influence social relations, both of which have been suggested to be important factors in the maintenance of NSSI (Brown et al., 2002; Durand \& Crimmins, 1988; Iwata et al., 1994; Nock \& Prinstein, 2004, 2005). Our narrow focus was intentional and necessary in this case given the relative lack of systematic research currently available on NSSI and difficulties associated 
with recruiting adolescent self-injurers for laboratory-based studies. It is necessary for future research in this area to examine how the constructs examined in this study interact with each other and how they might interact with other factors to produce and maintain this prevalent and dangerous behavior problem.

\section{References}

Barlow, D. H., \& Craske, M. G. (2000). Mastery of your anxiety and panic: Client workbook for anxiety and panic (MAP 3). San Antonio, TX: Graywind/Psychological Corporation.

Baumeister, R. F. (1990). Suicide as escape from self. Psychological Review, 97(1), 90-113.

Biggam, F. H., \& Power, K. G. (1999). Suicidality and the state-trait debate on problem-solving deficits: A re-examination with incarcerated young offenders. Archives of Suicide Research, 5(1), 27-42.

Briere, J., \& Gil, E. (1998). Self-mutilation in clinical and general population samples: Prevalence, correlates, and functions. American Journal of Orthopsychiatry, 68(4), 609-620.

Brown, M. Z., Comtois, K. A., \& Linehan, M. M. (2002). Reasons for suicide attempts and nonsuicidal self-injury in women with borderline personality disorder. Journal of Abnormal Psychology, 111(1), 198202.

Chapman, A. L., Gratz, K. L., \& Brown, M. Z. (2006). Solving the puzzle of deliberate self-harm: The experiential avoidance model. Behaviour Research and Therapy, 44(3), 371-394.

Crick, N. R., \& Dodge, K. A. (1994). A review and reformulation of social information-processing mechanisms in children's social adjustment. Psychological Bulletin, 115, 74-101.

Crowell, S. E., Beauchaine, T. P., McCauley, E., Smith, C. J., Stevens, A. L., \& Sylvers, P. (2005). Psychological, autonomic, and serotonergic correlates of parasuicide among adolescent girls. Development and Psychopathology, 17(4), 1105-1127.

Dawson, M. E., Schell, A. M., \& Filion, D. L. (2000). The electrodermal system. In J. T. Cacioppo \& G. G. Berntson (Eds.), Handbook of psychophysiology (pp. 200-223). Cambridge, England: Cambridge University Press.

Dodge, K. A., \& Frame, C. L. (1982). Social cognitive biases and deficits in aggressive boys. Child Development, 53(3), 620-635.

Dodge, K. A., \& Somberg, D. R. (1987). Hostile attributional biases among aggressive boys are exacerbated under conditions of threats to the self. Child Development, 58(1), 213-224.

Donegan, N. H., Sanislow, C. A., Blumberg, H. P., Fulbright, R. K., Lacadie, C., Skudlarski, P., et al. (2003). Amygdala hyperreactivity in borderline personality disorder: Implications for emotional dysregulation. Biological Psychiatry, 54(11), 1284-1293.

Durand, V. M., \& Crimmins, D. B. (1988). Identifying the variables maintaining self-injurious behavior. Journal of Autism and Developmental Disorders, 18(1), 99-117.

D'Zurilla, T. J., \& Nezu, A. M. (1990). Development and preliminary evaluation of the social problem-solving inventory. Psychological Assessment: A Journal of Consulting and Clinical Psychology, 2(2), 156163.

Ebner-Priemer, U. W., Badeck, S., Beckmann, C., Wagner, A., Feige, B., Weiss, I., et al. (2005). Affective dysregulation and dissociative experience in female patients with borderline personality disorder: A startle response study. Journal of Psychiatric Research, 39(1), 85-92.

Edman, G., Asberg, M., Levander, S., \& Schalling, D. (1986). Skin conductance habituation and cerebrospinal fluid 5-hydroxyindoleacetic acid in suicidal patients. Archives of General Psychiatry, 43(6), 586592.

Favazza, A. R. (1989). Why patients mutilate themselves. Hospital \& Community Psychiatry, 40(2), 137-145.

Favazza, A. R. (1996). Bodies under siege: Self-mutilation and body modification in culture and psychiatry (2nd ed.). Baltimore: Johns Hopkins University Press.

Glassman, L. H., Weierich, M. R., Hooley, J. M., Deliberto, T. L., \& Nock, M. K. (2007). Child maltreatment, non-suicidal self-injury, and the mediating role of self-criticism. Behaviour Research and Therapy, 45, 2483-2490.

Goddard, P., \& McFall, R. M. (1992). Decision-making skills and heterosocial competence in college women: An information-processing analysis. Journal of Social and Clinical Psychology, 11, 401-425.

Grant, D. A., \& Berg, E. A. (1948). A behavioral analysis of degree of impairment and ease of shifting to new responses in a Weigl-type card sorting problem. Journal of Experimental Psychology, 39, 404-411.

Gresham, F. M., \& Elliott, S. N. (1990). Social Skills Rating System manual. Circle Pines, MN: American Guidance Service.

Haines, J., Williams, C. L., Brain, K. L., \& Wilson, G. V. (1995). The psychophysiology of self-mutilation. Journal of Abnormal Psychology, 104(3), 471-489.

Hawton, K., Cole, D., O'Grady, J., \& Osborn, M. (1982). Motivational aspects of deliberate self-poisoning in adolescents. British Journal of Psychiatry, 141, 286-291.

Heaton, R. K., Chelune, G. J., Talley, J. L., Kay, G. G., \& Curtis, G. (1993). Wisconsin Card Sort Test (WCST) manual revised and expanded. Odessa, FL: Psychological Assessment Resources.

Herpertz, S. C., Dietrich, T. M., Wenning, B., Krings, T., Erberich, S. G., Willmes, K., et al. (2001). Evidence of abnormal amygdala functioning in borderline personality disorder: A functional MRI study. Biological Psychiatry, 50(4), 292-298.

Herpertz, S. C., Kunert, H. J., Schwenger, U. B., \& Sass, H. (1999). Affective responsiveness in borderline personality disorder: A psychophysiological approach. American Journal of Psychiatry, 156(10), $1550-1556$.

Herpertz, S. C., Werth, U., Lukas, G., Qunaibi, M., Schuerkens, A., Kunert, H. J., et al. (2001). Emotion in criminal offenders with psychopathy and borderline personality disorder. Archives of General Psychiatry, 58(8), $737-745$.

Hirito, D. S., \& Seligman, M. E. P. (1975). Generality of learned helplessness in man. Journal of Personality and Social Psychology, 31(2), 311-327.

Ingram, R. E. (Ed.). (1986). Information processing approaches to clinical psychology. San Diego, CA: Academic Press.

Iwata, B. A., Pace, G. M., Dorsey, M. F., Zarcone, J. R., Vollmer, T. R., Smith, R. G., et al. (1994). The functions of self-injurious behavior: An experimental-epidemiological analysis. Journal of Applied Behavior Analysis, 27(2), 215-240

Jaccard, J., \& Guilamo-Ramos, V. (2002). Analysis of variance frameworks in clinical child and adolescent psychology: Issues and recommendations. Journal of Clinical Child and Adolescent Psychology, 31(1), 130-146

Kaufman, J., Birmaher, B., Brent, D. A., Rao, U., \& Ryan, N. D. (1997). Schedule for Affective Disorders and Schizophrenia for School Age Children, Present and Lifetime Version (K-SADS-PL): Initial reliability and validity data. Journal of the American Academy of Child and Adolescent Psychiatry, 36, 980-988.

Kazdin, A. E., \& Nock, M. K. (2003). Delineating mechanisms of change in child and adolescent therapy: Methodological issues and research recommendations. Journal of Child Psychology and Psychiatry, 44(8), $1116-1129$.

Kazdin, A. E., Siegel, T. C., \& Bass, D. (1992). Cognitive problem-solving skills training and parent management training in the treatment of antisocial behavior in children. Journal of Consulting and Clinical Psychology, 60(5), 733-747.

Kehrer, C. A., \& Linehan, M. M. (1996). Interpersonal and emotional problem solving skills and parasuicide among women with borderline personality disorder. Journal of Personality Disorders, 10, 153-163. 
Kessler, R. C., Borges, G., \& Walters, E. E. (1999). Prevalence of and risk factors for lifetime suicide attempts in the National Comorbidity Survey. Archives of General Psychiatry, 56(7), 617-626.

Klonsky, E. D. (2007). The functions of deliberate self-injury: A review of the evidence. Clinical Psychology Review, 27(2), 226-239.

Klonsky, E. D., Oltmanns, T. F., \& Turkheimer, E. (2003). Deliberate self-harm in a nonclinical population: Prevalence and psychological correlates. American Journal of Psychiatry, 160(8), 1501-1508.

Linehan, M. M. (1993). Cognitive-behavioral treatment of borderline personality disorder. New York: Guilford Press.

Linehan, M. M., Armstrong, H. E., Suarez, A., Allmon, D., \& Heard, H. L. (1991). Cognitive-behavioral treatment of chronically parasuicidal borderline patients. Archives of General Psychiatry, 48(12), 1060-1064.

Linehan, M. M., Comtois, K. A., Murray, A. M., Brown, M. Z., Gallop, R. J., Heard, H. L., et al. (2006). Two-year randomized controlled trial and follow-up of dialectical behavior therapy vs. therapy by experts for suicidal behaviors and borderline personality disorder. Archives of General Psychiatry, 63(7), 757-766.

Lloyd, E., Kelley, M. L., \& Hope, T. (1997, April). Self-mutilation in a community sample of adolescents: Descriptive characteristics and provisional prevalence rates. Paper presented at the meeting of the Society for Behavioral Medicine, New Orleans, LA.

Lynch, T. R., Chapman, A. L., Rosenthal, M. Z., Kuo, J. R., \& Linehan, M. M. (2006). Mechanisms of change in dialectical behavior therapy: Theoretical and empirical observations. Journal of Clinical Psychology, 62(4), 459-480.

MacKay, D. G., Shafto, M., Taylor, J. K., Marian, D. E., Abrams, L., \& Dyer, J. R. (2004). Relations between emotion, memory, and attention: Evidence from taboo Stroop, lexical decision, and immediate memory tasks. Memory and Cognition, 32(3), 474-488.

McFall, R. M. (1982). A review and reformulation of the concept of social skills. Behavioral Assessment, 4, 1-33.

Menninger, K. (1938). Man against himself. New York: Harcourt Brace World.

Miller, A. L., Rathus, J. H., \& Linehan, M. M. (2007). Dialectical behavior therapy with suicidal adolescents. New York: Guilford Press.

Mindware. (2005). EDA 2.1 [computer software]. Columbus, OH: Author.

Najmi, S., Wegner, D. M., \& Nock, M. K. (2007). Thought suppression and self-injurious thoughts and behaviors. Behaviour Research and Therapy, 45, 1957-1965.

Nisbett, R. E., \& Wilson, T. D. (1977). Telling more than we can know: Verbal reports on mental processes. Psychological Review, 84(3), 231-259.

Nock, M. K. (2003). Progress review of the psychosocial treatment of child conduct problems. Clinical Psychology: Science and Practice, 10(1), 1-28.

Nock, M. K. (2006). Social Problem Solving Skills Test: Administration and coding manual. Unpublished manuscript, Harvard University, Cambridge, MA.

Nock, M. K., Holmberg, E. B., Photos, V. I., \& Michel, B. D. (2007). The Self-Injurious Thoughts and Behaviors Interview: Development, reliability, and validity in an adolescent sample. Psychological Assessment, 19, 309-317.

Nock, M. K., Joiner, T. E., Jr., Gordon, K. H., Lloyd-Richardson, E., \& Prinstein, M. J. (2006). Non-suicidal self-injury among adolescents: Diagnostic correlates and relation to suicide attempts. Psychiatry Research, 144(1), 65-72.

Nock, M. K., \& Kazdin, A. E. (2002). Examination of affective, cognitive, and behavioral factors and suicide-related outcomes in children and young adolescents. Journal of Clinical Child and Adolescent Psychology, 31(1), 48-58.

Nock, M. K., \& Kessler, R. C. (2006). Prevalence of and risk factors for suicide attempts versus suicide gestures: Analysis of the National Comorbidity Survey. Journal of Abnormal Psychology, 115(3), 616-623.

Nock, M. K., \& Prinstein, M. J. (2004). A functional approach to the assessment of self-mutilative behavior. Journal of Consulting and Clinical Psychology, 72(5), 885-890.

Nock, M. K., \& Prinstein, M. J. (2005). Clinical features and behavioral functions of adolescent self-mutilation. Journal of Abnormal Psychology, 114(1), 140-146.

Nock, M. K., Wedig, M. M., Holmberg, E. B., \& Hooley, J. M. (in press). Emotion Reactivity Scale: Psychometric evaluation and relation to selfinjurious thoughts and behaviors. Behavior Therapy.

O'Carroll, P. W., Berman, A. L., Maris, R., \& Moscicki, E. (1996). Beyond the tower of Babel: A nomenclature for suicidology. Suicide and LifeThreatening Behavior, 26(3), 237-252.

Orbach, I., Rosenheim, E., \& Hary, E. (1987). Some aspects of cognitive functioning in suicidal children. Journal of the American Academy of Child and Adolescent Psychiatry, 26(2), 181-185.

Platt, J. J., Spivack, G., \& Bloom, W. (1975). Manual for the Means-End Problem-Solving (MEPS) Procedure: A measure of interpersonal problemsolving skill. Philadelphia: Hahnemann Medical College Hospital.

Pollock, L. R., \& Williams, J. M. (1998). Problem solving and suicidal behavior. Suicide and Life Threatening Behavior, 28(4), 375-387.

Pollock, L. R., \& Williams, J. M. (2001). Effective problem solving in suicide attempters depends on specific autobiographical recall. Suicide and Life Threatening Behavior, 31(4), 386-396.

Rodham, K., Hawton, K., \& Evans, E. (2004). Reasons for deliberate self-harm: Comparison of self-poisoners and self-cutters in a community sample of adolescents. Journal of the American Academy of Child and Adolescent Psychiatry, 43(1), 80-87.

Ross, S., \& Heath, N. (2002). A study of the frequency of self-mutilation in a community sample of adolescents. Journal of Youth and Adolescence, 31(1), 67-77.

Rudd, M. D., Joiner, T. E., \& Rajab, M. H. (2001). Treating suicidal behavior: An effective, time-limited approach. New York: Guilford Press.

Ruggero, C. J., \& Johnson, S. L. (2006). Reactivity to a laboratory stressor among individuals with bipolar I disorder in full or partial remission. Journal of Abnormal Psychology, 115(3), 539-544.

Sadowski, C., \& Kelley, M. L. (1993). Social problem solving in suicidal adolescents. Journal of Consulting and Clinical Psychology, 61(1), 121-127.

Schotte, D. E., \& Clum, G. A. (1987). Problem-solving skills in suicidal psychiatric patients. Journal of Consulting and Clinical Psychology, 55(1), 49-54.

Schotte, D. E., Cools, J., \& Payvar, S. (1990). Problem-solving deficits in suicidal patients: Trait vulnerability or state phenomenon? Journal of Consulting and Clinical Psychology, 58(5), 562-564.

Shapiro, S. S., \& Wilks, M. B. (1965). An analysis of variance test for normality. Biometrika, 52, 591-611.

Tabachnick, B. G., \& Fidell, L. S. (2001). Using multivariate statistics (4th ed.). Boston: Allyn and Bacon.

Wechsler, D. (1999). Wechsler Abbreviated Scale of Intelligence. San Antonio, TX: Psychological Corporation.

Whitlock, J., Eckenrode, J., \& Silverman, D. (2006). Self-injurious behaviors in a college population. Pediatrics, 117(6), 1939-1948.

Williams, J. M., Barnhofer, T., Crane, C., \& Beck, A. T. (2005). Problem solving deteriorates following mood challenge in formerly depressed patients with a history of suicidal ideation. Journal of Abnormal Psychology, 114(3), 421-431.

Zoroglu, S. S., Tuzun, U., Sar, V., Tutkun, H., Savacs, H. A., Ozturk, M., et al. (2003). Suicide attempt and self-mutilation among Turkish high school students in relation with abuse, neglect and dissociation. Psychiatry and Clinical Neurosciences, 57(1), 119-126.

Received February 6, 2007

Revision received July 23, 2007

Accepted July 26, 2007 